\title{
Integration of patient-reported outcome measures with key clinical outcomes after immediate latissimus dorsi breast reconstruction and adjuvant treatment
}

\author{
Z. E. Winters ${ }^{1}$, J. Haviland ${ }^{2}$, V. Balta ${ }^{1}$, J. Benson ${ }^{3}$, A. Reece-Smith ${ }^{1}$ and N. Betambeau ${ }^{1}$, on behalf of \\ the Prospective Trial Management Group*
}

${ }^{1}$ Breast Reconstruction Quality of Life and Clinical Outcomes Research Group, School of Clinical Sciences, University of Bristol and University Hospitals of Bristol NHS Foundation Trust, Bristol Royal Infirmary, Bristol, ${ }^{2}$ Institute of Cancer Research Clinical Trials and Statistics Unit (ICR-CTSU), Division of Clinical Studies, Institute of Cancer Research, Sutton, and ${ }^{3}$ Cambridge University Hospitals NHS Foundation Trust, Cambridge, UK

Correspondence to: Dr Z. E. Winters, Breast Reconstruction Quality of Life and Clinical Outcomes Research Group, School of Clinical Sciences, University of Bristol and University Hospitals of Bristol NHS Foundation Trust, Bristol Royal Infirmary, Upper Maudlin Street, Bristol BS2 8HW, UK (e-mail: zoe.winters@bristol.ac.uk)

Background: Clinical evidence on patient-reported outcome measures (PROMS) in breast reconstruction is lacking. The aim of this study was to evaluate PROMs in implant-assisted latissimus dorsi (LDI) or tissue-only autologous latissimus dorsi (ALD) flap reconstruction in relation to complications and adjuvant treatments.

Methods: This was a prospective cohort study involving six UK centres. Eligible patients had primary early-stage breast cancer. The European Organization for Research and Treatment of Cancer qualityof-life questionnaire (QLQ)-C30 and QLQ-BR23, Functional Assessment of Cancer Therapy - Breast Cancer scale (FACT-B), Body Image Scale, and Hospital Anxiety and Depression Scale were completed before operation and at 3, 6 and 12 months after surgery.

Results: A total of 182 patients (82 LDI and 100 ALD) were recruited between 2007 and 2010 with symptomatic (59.9 per cent) or screen-detected (39.6 per cent) cancers. Some $\mathbf{6 4 . 3}$ per cent had lymph node-negative disease; 30 per cent of the LDI group had radiotherapy, compared with 53.0 per cent in the ALD group $(P=0.004)$. Early complications up to 3 months after surgery were reported in 66 and 51.0 per cent of patients in the LDI and ALD groups respectively $(P=0.062)$ and long-term complications (4-12 months) in 48 and 45.0 per cent $(P=0.845)$. Role functioning and pain $(P=0.002$ for both) were adversely affected in the ALD group compared with results in the LDI group, with no significant effects of radiotherapy on any health-related quality of life (HRQL). Chemotherapy and early complications adversely affected HRQL, which improved between 3 and 12 months after surgery $(P<\mathbf{0 . 0 1 0}$ for all).

Conclusion: There is evidence of similar HRQL between types of latissimus dorsi breast reconstruction for up to a year after surgery. There appear to be no overarching effects for radiotherapy after mastectomy on the specific HRQL domains studied in the short term. The identification of variables that affect HRQL is important, including their integration into the analysis of PROMs.

*Members of the Prospective Trial Management Group are co-authors of this study and can found under the heading Collaborators

Paper accepted 29 August 2012

Published online in Wiley Online Library (www.bjs.co.uk). DOI: 10.1002/bjs.8959

\section{Introduction}

Earlier detection and improved treatments for breast cancer have yielded long-term survival rates of more than 80 per cent following initial diagnosis ${ }^{1}$. This has increased the focus on the impact of surgical and other treatments on health-related quality of life (HRQL $)^{2}$. Up to 40 per cent of women diagnosed annually with breast cancer in the UK undergo mastectomy ${ }^{3}$, which affects their quality of life 
in terms of psychosocial, physical, aesthetic, body image and sexual issues ${ }^{4,5}$. Indeed, more than half of women experience general health problems according to the UK National Mastectomy and Breast Reconstruction Audit ${ }^{3}$.

Whatever the potential psychological benefits, the UK National Institute for Health and Clinical Excellence (NICE) recommends that reconstruction be offered as an immediate procedure to all women who have had a mastectomy ${ }^{6}$. The UK breast reconstruction audit revealed that 21 per cent of women underwent immediate breast reconstruction between 2008 and 2009, compared with 11 per cent in $2005-2006^{3,6}$. Although a range of reconstructive surgery is practised, from simple subpectoral implant insertion to complex free flaps, a latissimus dorsi (LD)-based flap is one of the most widely offered procedures in the $\mathrm{UK}^{3}$. The UK breast reconstruction audit found that pedicled flaps, whether autologous or implant-assisted, constituted almost half of all immediate and delayed breast reconstructions, compared with free microvascular flaps ${ }^{3}$. The types of $\mathrm{LD}$ technique are well established; autologous breast reconstruction performed as an extended LD dissection is routine in many units $^{3,7}$.

Increasingly, decisions on adjuvant therapies influence recommendations for either immediate or delayed breast reconstruction ${ }^{7}$. Postmastectomy radiotherapy (PMRT) may confer a survival benefit when combined with systemic adjuvant therapies ${ }^{8}$; approximately 40 per cent of women in the $\mathrm{UK}$ receive $\mathrm{PMRT}^{3}$. Broadened indications for PMRT are modifying the practice of breast reconstruction surgery, in terms of both timing and type ${ }^{9}$. Complication rates following irradiation of implant-based reconstructions are high, with severe capsular contracture rates of 28 percent at 4 years ${ }^{7}$. Many surgeons are reluctant to recommend immediate implant-assisted LD reconstruction in this setting and favour either a delayed or a staged procedure ${ }^{7,9}$. By contrast, exclusively tissuebased autologous breast reconstruction is more tolerant of radiotherapy. There were no significant differences in patient self-reported cosmetic outcomes or body image between types of LD breast reconstruction, regardless of PMRT, at a median follow-up of 3 years ${ }^{7}$.

Clinical trials should incorporate outcomes from the perspective of patients: patient-reported outcome measures $(\mathrm{PROMs})^{10}$. Evidence should include the impact of an intervention on the patient in terms of HRQL, to assess net benefits fully ${ }^{5}$. Two systematic reviews on breast reconstruction and HRQL revealed the majority of studies to be flawed methodologically, with no data evaluating the effects of LD breast reconstruction on HRQL ${ }^{4,11}$.
Randomized clinical trials are controversial in the field of breast reconstruction. Prospective cohort studies potentially can recruit a larger and more representative sample of patients ${ }^{4,11,12}$. The present study was a pilot phase to a planned randomized controlled trial and was designed as hypothesis-generating, with no a priori primary outcome defined. A prospective longitudinal study was conducted in several centres to evaluate whether any differences in HRQL could be demonstrated in terms of the extent of donor-site tissue dissection following immediate LD breast reconstruction with, and without adjuvant chemotherapy and/or PMRT.

\section{Methods}

A prospective longitudinal cohort study was carried out (National Research Ethics Committee Wiltshire: 05/Q2008/14 approval) in six UK centres (Bristol, Cambridge, Glasgow, Hull, Swindon and York) with patients recruited between January 2007 and May 2010. Women with pathologically confirmed stage $0-\mathrm{II}$ breast cancer ${ }^{13}$, including ductal carcinoma in situ (DCIS), and women with bilateral disease were included in the study. Women with a previous breast cancer were excluded. Other contraindications to immediate breast reconstruction were applicable, such as surgical or medical co-morbidity, or metastatic disease.

Eligible women gave written informed consent after biopsy confirmation of breast cancer. Participants were assessed before surgery for the likelihood of PMRT according to local policy using information from breast magnetic resonance imaging (MRI) and/or sentinel lymph node biopsy ${ }^{14,15}$. MRI was used to determine tumour size, multicentricity, or proximity to pectoral muscle and skin. Women eligible for neoadjuvant chemotherapy, but who ultimately required mastectomy, were included. In accordance with the UK NICE guidelines, all women had fully informed discussions regarding the range of reconstructive options, likelihood of PMRT, comorbidities and patient preferences ${ }^{16}$. Informed consent was obtained on the part of all healthcare professionals, and was recommended according to national guidelines. The process regarding the surgical choice by the patient or the healthcare professionals across the six centres was beyond the scope of the study. HRQL questionnaire packs were handed directly to patients at the second preoperative visit (baseline), and subsequently posted with a self-addressed freepost envelope at 3, 6 and 12 months after surgery. Recurrent (local or distant) disease did not exclude questionnaire administration, except in the context of confirmed cerebral metastases. 


\section{Primary endpoints}

HRQL was assessed using validated self-reported patient measures to evaluate the impact of breast cancer and its treatment ${ }^{5,17,18}$. The European Organization for Research and Treatment of Cancer (EORTC) generic qualityof-life questionnaire QLQ-C30 was used ${ }^{19}$, comprising functional and symptom subscales. The EORTC breast cancer module (QLQ-BR23) was used to evaluate breast and arm symptoms ${ }^{19}$, and the ten-item Body Image Scale $(\mathrm{BIS})^{20}$ was employed as a measure of body image. The Hospital Anxiety and Depression Scale (HADS), consisting of two subscales to distinguish anxiety and depression, was used to evaluate the need for psychological referral ${ }^{21}$. Finally, the Functional Assessment of Cancer Therapy - Breast Cancer scale (FACT-B) quality-of-life questionnaire was used, which is designed for women with breast cancer and includes subscales assessing physical, social, emotional and functional wellbeing ${ }^{22}$. These PROMs were used in the absence of any other validated breast reconstruction-specific questionnaires available at the commencement of the study ${ }^{4,11}$.

\section{Secondary endpoints}

Patient response rates for questionnaire completion were documented at all time points ${ }^{18}$. Adverse events and complications were collected as per protocol on clinical record forms, and stratified by severity and timing after surgery using the classification system described by Dindo-Clavien ${ }^{23}$. Locoregional recurrence and distant metastatic disease were defined using established criteria $^{24}$.

\section{Patient characteristics}

Explanatory variables used in the study included age at diagnosis, menopausal status, mode of breast cancer detection, tumour characteristics, co-morbidities, treatments received (including surgery, chemotherapy, hormone therapy and radiotherapy) and sociodemographic factors $^{2,5,24}$.

\section{Follow-up}

Clinical follow-up was scheduled at 1 and 2 weeks after surgery, then at 3, 6 and 12 months from the date of operation. Early (up to and including 3 months) and longterm (from 4 to 12 months) postsurgical complications were documented, as well as any subsequent surgical interventions. Complications were graded as minor (level 1 , no specific intervention), moderate (level 2, requirement for antibiotics or blood transfusion) or severe (level 3, indication for surgery - local or general anaesthesia) ${ }^{23}$.

\section{Statistical analysis}

This was primarily a hypothesis-generating study, so the sample size was determined on the basis of obtaining sufficient numbers of women in the main treatment groups of interest for statistical analysis, rather than to test a specific a priori hypothesis. The four treatment groups of interest (with the expected proportions in each group) were LD implant without radiotherapy (60 per cent), LD implant with radiotherapy (10 per cent), extended autologous LD without radiotherapy (20 per cent) and extended autologous LD with radiotherapy $(10 \text { per cent })^{3,7}$. A target of at least 150 women for the total study size was set, with the aim of providing a minimum of 15 patients within each category.

Demographic and clinical data were compared between the surgical groups using the $\chi^{2}$ test, $\chi^{2}$ test for trend or Fisher's exact test where appropriate, for categorical variables. Quantitative data were summarized using the mean and standard deviation (s.d.) or the median and interquartile range (i.q.r.) where distributions were skewed, and compared between surgical groups using the $t$ test or Mann-Whitney $U$ test respectively. Rates of specific types of early and long-term complications were not compared formally between surgical groups, as numbers in some categories were small. HRQL subscales were summarized using the median (i.q.r.) as distributions were skewed. The 3- and 12-month time points were selected for graphical presentation of the HRQL subscales as these were considered to represent short- and longterm outcomes for the study. Longitudinal analysis was done by fitting a separate generalized estimating equations model for each HRQL subscale, which included all of the following potential predictors: type of surgery, radiotherapy, chemotherapy, early (3 months or less) and long-term (4-12 months) complications, age at operation, time point (3, 6 or 12 months) and baseline (before surgery) HRQL score. To take account of the many significance tests performed, a more conservative cut-off was taken to indicate statistical significance $(P \leq 0.010)$. The statistical program SPSS $^{\circledR}$ version 19 (IBM, Armonk, New York, USA) was used for statistical analysis.

\section{Results}

Of the 236 women assessed for eligibility, 182 were recruited; 82 (45.1 per cent) had implant-assisted latissimus dorsi (LDI) and 100 (54.9 per cent) had autologous 


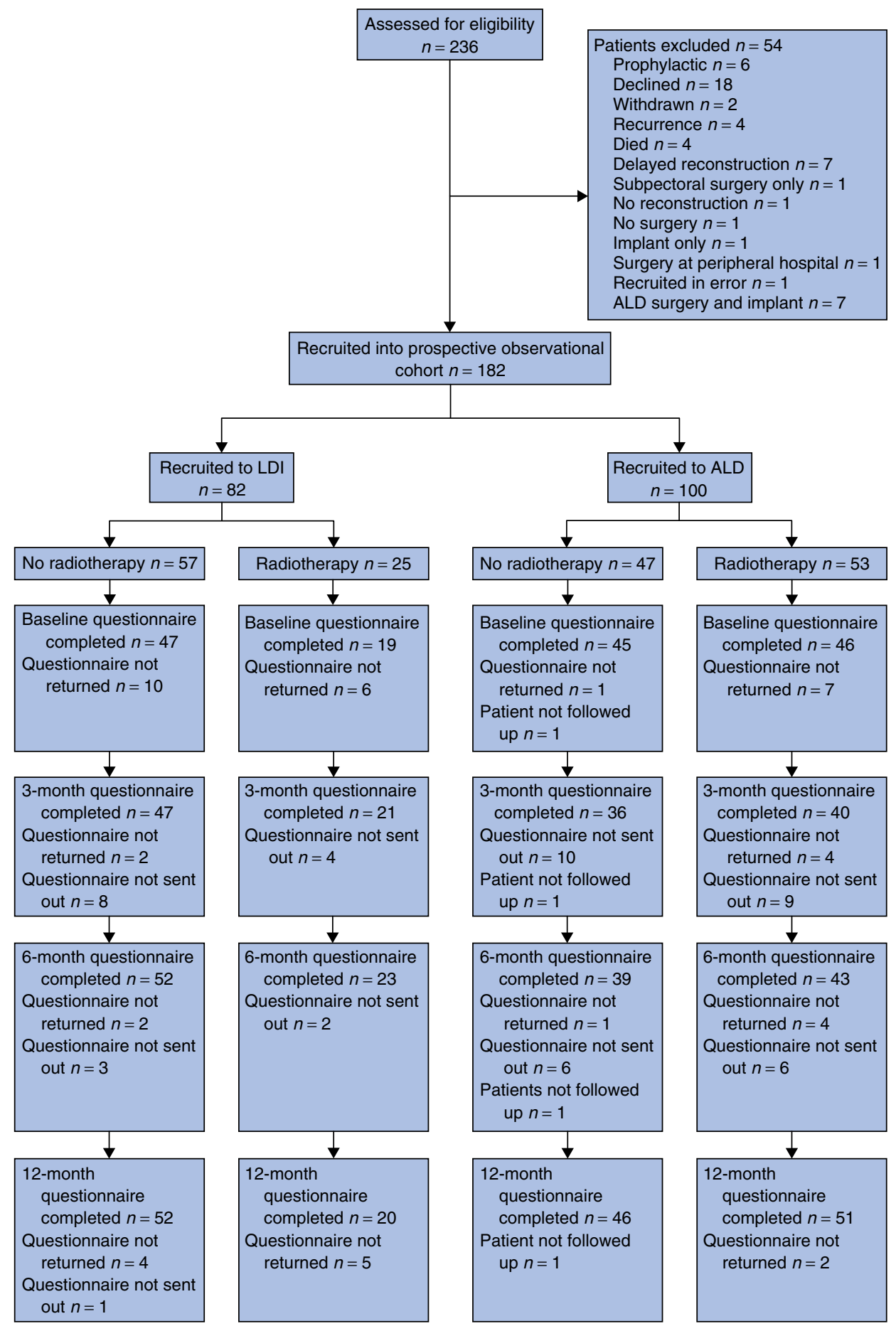

Fig. 1 Progress of participants through the study. The numbers of questionnaires not sent to patients and patients not followed up relate to administrative error and do not reflect recurrent disease. Questionnaires unreturned by patients are in keeping with protocol-defined reminder letters and contact with patients. ALD, autologous latissimus dorsi breast reconstruction; LDI, implant-assisted latissimus dorsi breast reconstruction 
Table 1 Comparison of baseline demographic and clinical characteristics in women having implant-assisted or extended latissimus dorsi breast reconstruction

\begin{tabular}{|c|c|c|c|c|}
\hline & & LDI $(n=82)$ & $\operatorname{ALD}(n=100)$ & $P \S$ \\
\hline Age (years) ${ }^{\star}$ & & $51 \cdot 1(9.5)$ & $49 \cdot 4(8 \cdot 3)$ & $0 \cdot 187$ पा \\
\hline Body mass index $\left(\mathrm{kg} / \mathrm{m}^{2}\right)^{\star}$ & & $25 \cdot 2(4 \cdot 0)$ & $27 \cdot 4(4 \cdot 8)$ & $0.001 \Phi$ \\
\hline \multirow[t]{5}{*}{ Highest educational level } & $\begin{array}{l}\text { No formal qualifications/vocational } \\
\text { qualifications only }\end{array}$ & $8(10)$ & $19(19.0)$ & 0.023\# \\
\hline & Ordinary level/GCSE & $29(35)$ & $36(36 \cdot 0)$ & \\
\hline & Advanced level & $11(13)$ & $17(17 \cdot 0)$ & \\
\hline & University degree/postgraduate & $31(38)$ & $22(22 \cdot 0)$ & \\
\hline & Unknown & $3(4)$ & $6(6 \cdot 0)$ & \\
\hline \multirow[t]{3}{*}{ Employed } & No & $30(37)$ & $22(22 \cdot 0)$ & 0.050 \\
\hline & Yes & $52(63)$ & $77(77 \cdot 0)$ & \\
\hline & Unknown & $0(0)$ & $1(1 \cdot 0)$ & \\
\hline \multirow[t]{2}{*}{ Other medical illnesses } & No & $69(84)$ & $91(91.0)$ & 0.237 \\
\hline & Yes & $13(16)$ & $9(9.0)$ & \\
\hline \multirow{4}{*}{$\begin{array}{l}\text { Previous breast or axillary } \\
\text { surgery }\end{array}$} & None & $44(54)$ & $58(58.0)$ & 0.401 \\
\hline & Wide local excision & $25(30)$ & $24(24.0)$ & \\
\hline & Other (including SLN-assisted sample, SLN) & $6(7)$ & $12(12.0)$ & \\
\hline & Unknown & $7(9)$ & $6(6 \cdot 0)$ & \\
\hline \multirow[t]{2}{*}{ Neoadjuvant chemotherapy } & No & $79(96)$ & $83(83.0)$ & 0.009 \\
\hline & Yes & $3(4)$ & $17(17 \cdot 0)$ & \\
\hline \multirow[t]{3}{*}{ Reason for surgery } & Screen-detected & $37(45)$ & $35(35.0)$ & 0.191 \\
\hline & Symptomatic & $44(54)$ & $65(65.0)$ & \\
\hline & Unknown & $1(1)$ & $0(0)$ & \\
\hline \multirow[t]{5}{*}{ Pattern of mastectomy } & NAC excision & $73(89)$ & $89(89.0)$ & $0.960 \dagger i$ \\
\hline & Nipple-sparing & $3(4)$ & $1(1 \cdot 0)$ & \\
\hline & Reduction mammoplasty & $3(4)$ & $7(7 \cdot 0)$ & \\
\hline & Standard mastectomy & $1(1)$ & $2(2 \cdot 0)$ & \\
\hline & Unknown & $2(2)$ & $1(1.0)$ & \\
\hline $\begin{array}{l}\text { Dry weight of mastectomy } \\
\text { specimen }(\mathrm{g}) \dagger\end{array}$ & & $\begin{array}{c}432(261-573 \\
90-1860)\end{array}$ & $\begin{array}{c}480(349-642 \\
134-1797)\end{array}$ & $0.047^{\star \star}$ \\
\hline \multirow[t]{6}{*}{ Type of axillary surgery } & None & 7 (9) & $6(6 \cdot 0)$ & $<0.001$ \\
\hline & Level 1 axillary clearance & $16(20)$ & $2(2 \cdot 0)$ & \\
\hline & Level 2/3 axillary clearance & $31(38)$ & $56(56 \cdot 0)$ & \\
\hline & Axillary lymph node sample & $16(20)$ & $14(14.0)$ & \\
\hline & SLN-assisted sample & $12(15)$ & $21(21.0)$ & \\
\hline & Unknown & $0(0)$ & $1(1 \cdot 0)$ & \\
\hline \multirow[t]{4}{*}{ Closure of back wound } & Standard two-layer & $25(30)$ & $29(29.0)$ & 0.026 \\
\hline & Quilting & $37(45)$ & $59(59.0)$ & \\
\hline & Other: & $20(24)$ & $10(10.0)$ & \\
\hline & Unknown & $0(0)$ & $2(2 \cdot 0)$ & \\
\hline
\end{tabular}

Values in parentheses are percentages unless indicated otherwise; *values are mean(s.d.), and †values are median (interquartile range, range) for 68 and 85 patients with data on dry weight of mastectomy specimen in implant-assisted latissimus dorsi breast reconstruction (LDI) and autologous latissimus dorsi breast reconstruction (ALD) groups respectively. \$For example fibrin sealant or triamcinolone. GCSE, General Certificate of Secondary Education; NAC, nipple-areolar complex; SLN, sentinel lymph node. $\S \chi^{2}$ test, except $\mathbb{I} t$ test, \# $\chi^{2}$ test for trend and ${ }^{* *}$ Mann-Whitney $U$ test; $\dagger \dagger N A C$ excision versus all others (unknown categories were excluded from significance tests).

latissimus dorsi (ALD) breast reconstruction procedures (Fig. 1). Sociodemographic characteristics were similar between the surgical groups (Table 1), with a mean patient age of 50 years. A significantly higher mean body mass index was evident among women in the ALD compared with the LDI group (27.4 versus $25.2 \mathrm{~kg} / \mathrm{m}^{2}$; $P=0.001)$. Clinical characteristics were similar between the surgical groups for many of the assessed criteria, such as menopausal, marital, educational, employment and current smoking status, including other medical illnesses (Table 1; data not shown). Some 87.9 per cent of women had no significant co-morbidities; 26.9 per cent of women had undergone a recent therapeutic wide local excision, which subsequently required completion mastectomy to achieve surgical margin clearance. Baseline HRQL data for this group were comparable to those in patients undergoing mastectomy as the first procedure, although role functioning and arm symptoms were notably worse when mastectomy followed previous wide local excision (data not shown). Similar distributions of need 
Table 2 Comparison of pathology and adjuvant treatment in women having implant-assisted or extended latissimus dorsi breast reconstruction

\begin{tabular}{|c|c|c|c|c|}
\hline & & LDI $(n=82)$ & $\operatorname{ALD}(n=100)$ & $P \mathbb{I}$ \\
\hline \multirow[t]{4}{*}{ Pathology $\dagger$} & Invasive breast cancer only & $33(40)$ & $29(29.0)$ & 0.020 \\
\hline & DCIS only & $22(27)$ & $17(17 \cdot 0)$ & \\
\hline & Invasive + DCIS & $27(33)$ & $53(53.0)$ & \\
\hline & Unknown & $0(0)$ & $1(1 \cdot 0)$ & \\
\hline \multirow[t]{2}{*}{ Tumour size $(\mathrm{mm})^{\star} \ddagger$} & Invasive & $19(12-22,2-70)$ & $20(13-29,2-100)$ & $0.152 \#$ \\
\hline & DCIS & $32(15-50,1-85)$ & $31.5(17-45,1-120)$ & $0.924 \#$ \\
\hline \multirow[t]{4}{*}{ Grade (invasive) } & 1 & $15(25)$ & $10(12)$ & $0.111^{\star \star}$ \\
\hline & 2 & $29(48)$ & $40(49)$ & \\
\hline & 3 & $15(25)$ & $29(35)$ & \\
\hline & Unknown & $1(2)$ & $3(4)$ & \\
\hline \multirow[t]{2}{*}{ Multifocal } & No & $37(45)$ & $51(51 \cdot 0)$ & 0.522 \\
\hline & Yes & $45(55)$ & $49(49.0)$ & \\
\hline \multirow[t]{3}{*}{ Lymphovascular invasion } & No & $60(73)$ & $55(55.0)$ & 0.019 \\
\hline & Yes & $19(23)$ & $40(40 \cdot 0)$ & \\
\hline & Unknown & $3(4)$ & $5(5 \cdot 0)$ & \\
\hline \multirow[t]{2}{*}{ Margin positivity $(<1 \mathrm{~mm}) \S$} & Invasive & 13 of $59(22)$ & 4 of $79(5)$ & 0.006 \\
\hline & DCIS & 8 of $47(17)$ & 13 of $66(20)$ & 0.908 \\
\hline \multirow[t]{2}{*}{ Lymph node status* } & No. of nodes removed & $8(5-12,0-22)$ & $9(5-14,0-36)$ & $0.297 \#$ \\
\hline & No. of involved nodes & $0(0-1,0-14)$ & $0(0-2,0-26)$ & $0.016 \#$ \\
\hline \multirow[t]{2}{*}{ Lymph node positivity } & Yes & $22(27)$ & $43(43.0)$ & 0.035 \\
\hline & No & $60(73)$ & $57(57 \cdot 0)$ & \\
\hline \multirow[t]{2}{*}{ Postmastectomy chemotherapy } & No & $45(55)$ & $51(51 \cdot 0)$ & 0.710 \\
\hline & Yes & $37(45)$ & $49(49.0)$ & \\
\hline \multirow[t]{4}{*}{ Herceptin } & No & $27(33)$ & $26(26 \cdot 0)$ & 0.541 \\
\hline & Yes & $7(9)$ & $11(11.0)$ & \\
\hline & Unknown & $2(2)$ & $1(1.0)$ & \\
\hline & NA (HER2 negative) & $46(56)$ & $62(62.0)$ & \\
\hline \multirow[t]{5}{*}{ Hormone therapy } & None & $10(12)$ & $14(14.0)$ & 0.283 \\
\hline & Tamoxifen & $41(50)$ & $39(39.0)$ & \\
\hline & Aromatase inhibitor & $15(18)$ & $26(26.0)$ & \\
\hline & Unknown & $2(2)$ & $1(1 \cdot 0)$ & \\
\hline & NA (oestrogen receptor-negative) & $14(17)$ & $20(20.0)$ & \\
\hline \multirow[t]{2}{*}{ Postmastectomy radiotherapy } & No & $57(70)$ & $47(47 \cdot 0)$ & 0.004 \\
\hline & Yes & $25(30)$ & $53(53.0)$ & \\
\hline
\end{tabular}

Values in parentheses are percentages unless indicated otherwise; *values are median (interquartile range, range). $\dagger$ Thus there were 60 cases of invasive cancer in the implant-assisted latissimus dorsi breast reconstruction (LDI) group and 82 cases in the autologous latissimus dorsi breast reconstruction (ALD) group, and 49 and 70 cases of ductal carcinoma in situ (DCIS) in the LDI and ALD groups respectively. $¥$ Tumour size was unknown for one invasive case in the ALD group and one DCIS case in the LDI group. §Margin status was unknown for one and three cases of invasive cancer, and for two and four cases of DCIS in the LDI and ALD groups respectively. NA, not applicable; HER2, human epidermal growth factor receptor 2. II $\chi^{2}$ test, except \#Mann-Whitney $U$ test, ${ }^{*} \chi^{2}$ test for trend (unknown and NA categories were excluded from significance tests).

for completion mastectomy between the surgical groups precluded any formal correction for this variable. Patients in the ALD group were more likely to have received neoadjuvant chemotherapy than those in the LDI group (17.0 versus 4 per cent respectively; $P=0.009)$.

Few women were current users of hormone replacement therapy or bisphosphonates (data not shown). The indications for mastectomy were similar in the two groups and a contralateral reduction mammoplasty was seldom necessary in either group (4 per cent for LDI versus 7 per cent for ALD). Axillary lymph node dissection was done more often in the ALD compared with the LDI group (56.0 versus 38 per cent respectively; $P<0.001$ ), in accordance with histopathology findings (Tables 1 and 2). Established and newer interventions were used to reduce donor-site seromas ${ }^{24}$.

\section{Histopathology, treatments and complications}

Patients included those with invasive carcinoma (34.1 per cent), extensive DCIS (21.4 per cent) and combined pathology ( 44.0 per cent) (Table 2). The median tumour size was $20 \mathrm{~mm}$ for the invasive component. More aggressive tumour characteristics were evident in the ALD group, with higher-grade tumours, more frequent lymphovascular invasion and higher rates of lymph node positivity, although the differences were not statistically significant. The significantly greater margin positivity 
rate of 22 percent for invasive disease in the LDI group, compared with 5 per cent in the ALD group, is largely inexplicable and would necessitate consideration of $\mathrm{PMRT}^{14,15}$. There were no differences between the groups for hormone receptors and human epidermal growth factor receptor (HER2) (data not shown). Adjuvant chemotherapy was administered to 47.3 per cent of women, and was similar in both groups, but PMRT was more common in women undergoing ALD (53.0 per cent versus 30 per cent for LDI; $P=0.004$ ) (Table 2). There were no differences between groups for adjuvant endocrine or herceptin treatment (data not shown). The numbers of patients with local and systemic recurrence were comparable between the groups, and were limited to a total of two and three patients respectively, with no deaths within 12 months.

Patients in the LDI and ALD groups had similar levels of early complications up to 3 months after surgery (66 versus 51.0 per cent respectively), and of long-term complications from 4 to 12 months (48 versus 45.0 per cent respectively) (Table 3).

Table 3 Comparison of early and late complications after surgery in women having implant-assisted or extended latissimus dorsi breast reconstruction

\begin{tabular}{|c|c|c|c|c|}
\hline & & LDI $(n=82)$ & $\operatorname{ALD}(n=100)$ & $P \S$ \\
\hline \multicolumn{5}{|l|}{ Early complications (0-3 months) } \\
\hline \multirow[t]{2}{*}{ Any early complication } & None & $28(34)$ & $49(49 \cdot 0)$ & 0.062 \\
\hline & Yes & $54(66)$ & $51(51.0)$ & \\
\hline \multirow[t]{3}{*}{ Highest level $\dagger$} & Minor & 17 of $54(31)$ & 21 of $51(41)$ & 0.116 \\
\hline & Moderate & 12 of $54(22)$ & 15 of $51(29)$ & \\
\hline & Severe & 25 of $54(46)$ & 15 of $51(29)$ & \\
\hline \multirow[t]{6}{*}{ Breast } & Infection & 8 of $54(15)$ & 4 of $51(8)$ & \\
\hline & Fat necrosis & 7 of $54(13)$ & 8 of $51(16)$ & \\
\hline & Skin necrosis & 23 of $54(43)$ & 11 of $51(22)$ & \\
\hline & Haematoma & 2 of $54(4)$ & 2 of $51(4)$ & \\
\hline & Implant infection or displacement & 6 of $54(11)$ & NA & \\
\hline & $\begin{array}{l}\text { Other (nipple loss, dehiscence, lymphoedema of } \\
\text { breast or arm) }\end{array}$ & 5 of $54(9)$ & 2 of $51(4)$ & \\
\hline \multirow[t]{5}{*}{ Back } & Dehiscence & 9 of $54(17)$ & 3 of $51(6)$ & \\
\hline & Infection & 5 of $54(9)$ & 8 of $51(16)$ & \\
\hline & Seroma ( $>5$ aspirations) & 22 of $54(41)$ & 25 of $51(49)$ & \\
\hline & Skin necrosis & 17 of $54(31)$ & 21 of $51(41)$ & \\
\hline & Other (loculated seroma, back pain, haematoma) & 9 of $54(17)$ & 10 of $51(20)$ & \\
\hline \multirow[t]{2}{*}{ Reoperation } & No & $32(39)$ & $35(35 \cdot 0)$ & 0.685 \\
\hline & Yes & $50(61)$ & $65(65.0)$ & \\
\hline \multirow[t]{4}{*}{ Reason for reoperation } & Complications & 24 of $50(48)$ & 20 of $65(31)$ & \\
\hline & To achieve symmetry/improve cosmetic appearance $\ddagger$ & 16 of $50(32)$ & 22 of $65(34)$ & \\
\hline & Nipple reconstruction & 28 of $50(56)$ & 49 of $65(75)$ & \\
\hline & Other (including prophylactic mastectomy) & 7 of $50(14)$ & 2 of $65(3)$ & \\
\hline \multicolumn{5}{|c|}{ Long-term complications (4-12 months) } \\
\hline \multirow[t]{2}{*}{ Any long-term complication } & None & $43(52)$ & $55(55 \cdot 0)$ & 0.845 \\
\hline & Yes & $39(48)$ & $45(45 \cdot 0)$ & \\
\hline \multirow[t]{3}{*}{ Highest level $\dagger$} & Minor & 14 of $39(36)$ & 27 of $45(60)$ & 0.042 \\
\hline & Moderate & 6 of $39(15)$ & 4 of $45(9)$ & \\
\hline & Severe & 19 of $39(49)$ & 14 of $45(31)$ & \\
\hline \multirow[t]{7}{*}{ Complications } & Capsular contracture & 6 of $39(15)$ & 0 of $45(0)$ & \\
\hline & Lymphoedema, breast & 4 of $39(10)$ & 7 of $45(16)$ & \\
\hline & Restriction of arm/shoulder movement & 6 of $39(15)$ & 5 of $45(11)$ & \\
\hline & Dog ear of any wound & 8 of $39(21)$ & 16 of $45(36)$ & \\
\hline & Implant complications & 5 of $39(13)$ & NA & \\
\hline & Back symptoms (e.g. stiffness, tightness, discomfort) & 6 of $39(15)$ & 13 of $45(29)$ & \\
\hline & $\begin{array}{l}\text { Other complications (including lymphoedema of arm, } \\
\text { loss of breast volume, asymmetry) }\end{array}$ & 17 of $39(44)$ & 14 of $45(31)$ & \\
\hline
\end{tabular}

Values in parentheses are percentages. $\nmid$ Dindo-Clavien classification of surgical complications ${ }^{23}$ : minor (level 1$)$, no pharmacological treatments as deviation from normal postoperative course; moderate (level 2), pharmacological treatments for complications (antibiotics, blood transfusions); severe (level 3), surgical intervention, either local or general anaesthetic. $¥$ Excluding nipple reconstruction. LDI, implant-assisted latissimus dorsi breast reconstruction; ALD, autologous latissimus dorsi breast reconstruction; NA, not applicable. $\S \chi^{2}$ test, except II $\chi^{2}$ test for trend (unknown categories were excluded from significance tests). 


\section{Patient-reported outcome measures and health-related quality of life}

Patient response rates for questionnaire completion were 86.3 per cent at baseline and 92.9 per cent at 12 months, with similar rates between the treatment groups (Fig. 1).

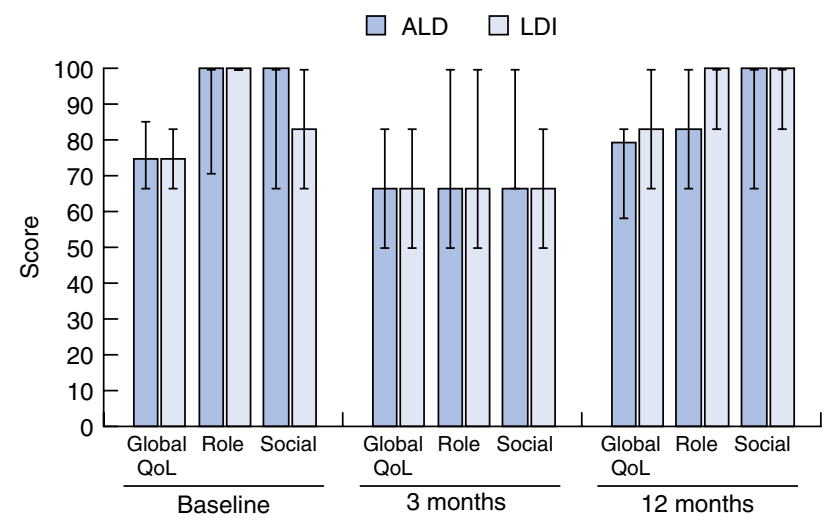

a Global QoL and functioning domains on the EORTC QLQ-C30

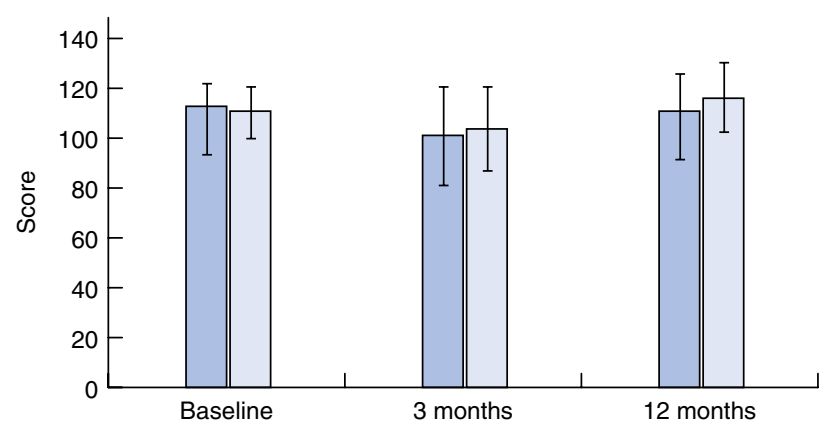

C Total score on FACT-B
Patterns of distributions of HRQL outcomes were similar between the surgery groups over time, with many of the outcomes from EORTC QLQ-C30 and QLQ-BR23 worsening from baseline to 3 months, and subsequently improving by 12 months (Fig. 2).

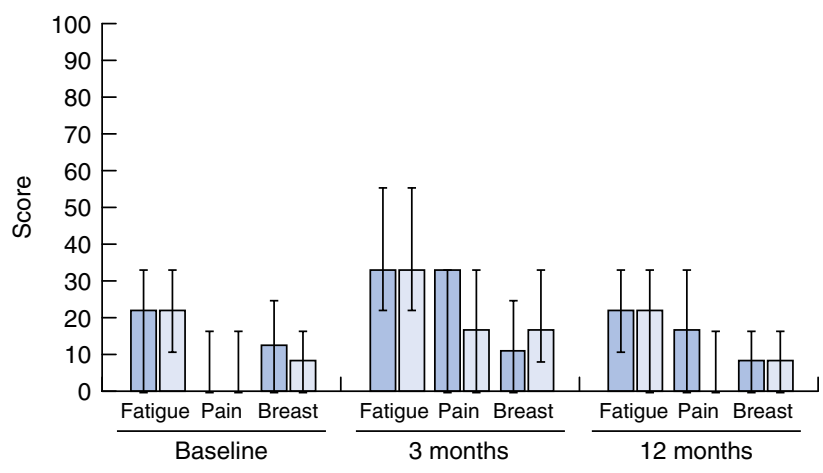

b Fatigue, pain and breast symptoms on the EORTC QLQ-C30 and QLQ-BR23

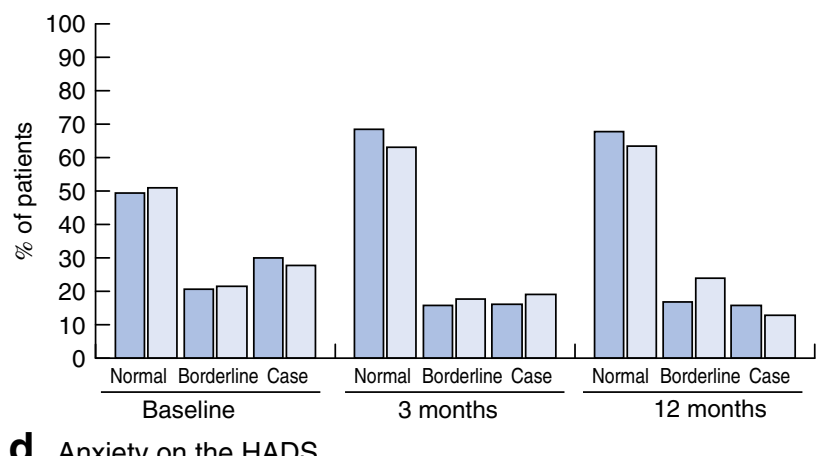

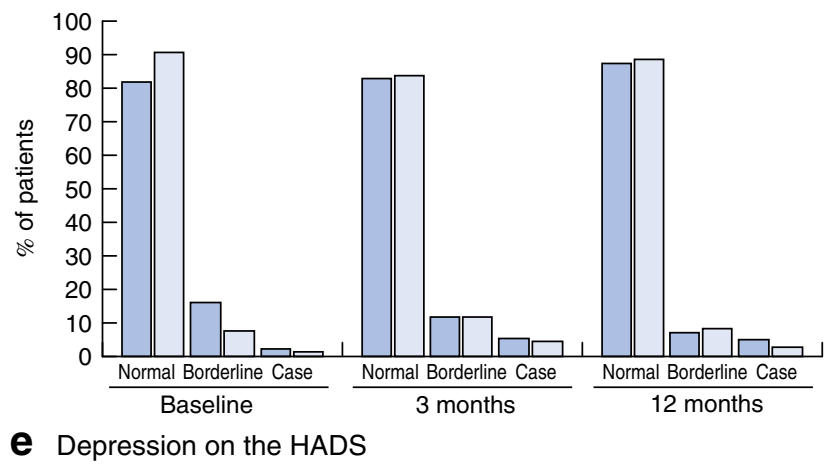

Fig. 2 Distribution of health-related quality-of-life (QoL) outcomes up to 1 year after implant-assisted latissimus dorsi (LDI) or autologous latissimus dorsi (ALD) breast reconstruction. a Median (interquartile range, i.q.r.) score for global QoL, and for role and social functioning domains on the European Organization for Research and Treatment of Cancer (EORTC) cancer generic quality-of-life questionnaire (QLQ-C30; range 0-100). b Median (i.q.r.) score for fatigue, pain and breast symptoms on the EORTC QLQ-C30 and the breast cancer module QLQ-BR23 (range 0-100). c Median (i.q.r.) total score on the Functional Assessment of Cancer Therapy - Breast Cancer scale (FACT-B; range 0-144). d Anxiety and e depression on the Hospital Anxiety and Depression Scale (HADS; range 0-21: 0-7, normal; 8-10, borderline; 11 or more, case) 
Table 4 Results of generalized estimating equation models investigating clinical and demographic predictors of health-related quality of life 3-12 months after breast reconstruction surgery

\begin{tabular}{|c|c|c|c|c|c|c|c|}
\hline \multirow[b]{2}{*}{ HRQL subscale } & \multicolumn{7}{|c|}{ Predictor of worse HRQL ( $P$ value) } \\
\hline & $\begin{array}{l}\text { Surgery (ALD } \\
\text { versus LDI) }\end{array}$ & Radiotherapy & Chemotherapy & $\begin{array}{c}\text { Early } \\
\text { complications }^{*}\end{array}$ & $\begin{array}{c}\text { Long-term } \\
\text { complications }^{*}\end{array}$ & $\begin{array}{l}\text { Younger } \\
\text { age }\end{array}$ & $\begin{array}{l}\text { Less time } \\
\text { since surgery }\end{array}$ \\
\hline \multicolumn{8}{|c|}{ EORTC QLQ-C30 and QLQ-BR23 } \\
\hline Global quality of life & 0.195 & 0.457 & $<0.001$ & $<0.001$ & 0.158 & 0.302 & $<0.001$ \\
\hline Role functioning & 0.002 & 0.330 & 0.026 & $<0.001$ & 0.684 & 0.278 & $<0.001$ \\
\hline Social functioning & 0.823 & 0.563 & 0.001 & 0.001 & 0.355 & 0.020 & $<0.001$ \\
\hline Fatigue & 0.285 & 0.311 & 0.006 & $<0.001$ & 0.814 & 0.326 & $<0.001$ \\
\hline Pain & 0.002 & 0.687 & 0.091 & 0.001 & $>0.99$ & 0.671 & $<0.001$ \\
\hline Breast symptoms & 0.800 & 0.487 & 0.929 & $<0.001$ & 0.014 & 0.147 & 0.009 \\
\hline Arm symptoms & 0.103 & 0.216 & 0.360 & 0.341 & 0.074 & 0.969 & 0.091 \\
\hline Body Image Scale & 0.491 & 0.983 & 0.891 & 0.079 & $<0.001$ & 0.021 & 0.079 \\
\hline \multicolumn{8}{|l|}{ HADS } \\
\hline Anxiety & 0.279 & 0.373 & 0.183 & 0.083 & 0.367 & 0.012 & 0.587 \\
\hline Depression & 0.236 & 0.572 & 0.035 & $<0.001$ & 0.826 & 0.368 & 0.002 \\
\hline \multicolumn{8}{|l|}{ FACT-B } \\
\hline Physical wellbeing & 0.076 & 0.033 & 0.060 & 0.011 & 0.877 & 0.330 & $<0.001$ \\
\hline Social wellbeing & 0.290 & 0.560 & 0.438 & 0.374 & 0.558 & 0.360 & 0.337 \\
\hline Emotional wellbeing & 0.870 & 0.018 & 0.474 & 0.158 & 0.227 & 0.209 & 0.040 \\
\hline Functional wellbeing & 0.485 & 0.574 & $<0.001$ & $<0.001$ & 0.844 & 0.619 & $<0.001$ \\
\hline Breast cancer subscale & 0.813 & 0.333 & 0.002 & 0.574 & 0.090 & 0.480 & $<0.001$ \\
\hline FACT-B total score & 0.519 & 0.215 & $<0.001$ & 0.011 & 0.468 & 0.493 & $<0.001$ \\
\hline
\end{tabular}

*Early (0-3 months) and long-term (4-12 months) complications graded by the Dindo-Clavien classification ${ }^{23}$ as none, minor, moderate or severe. HRQL, health-related quality of life; ALD, autologous latissimus dorsi breast reconstruction; LDI, implant-assisted latissimus dorsi breast reconstruction. EORTC QLQ, European Organization for the Research and Treatment of Cancer quality-of-life questionnaire; HADS, Hospital Anxiety and Depression Scale; FACT-B, Functional Assessment of Cancer Therapy- Breast Cancer scale. Separate models were fitted for each HRQL subscale including all predictors listed in the table and the baseline value of the HRQL subscale. $P<0.010$ indicates that the predictor was significantly associated with worse levels of the HRQL subscale.

Among the HRQL measures, only two domains from the EORTC QLQ-C30 (role functioning and pain) were significantly worsened after ALD compared with LDI reconstruction $(P=0.002$ for both). HRQL was not significantly different according to PMRT or age. Chemotherapy (adjuvant and neoadjuvant) significantly impaired global quality of life $(P<0.001)$, social functioning $(P=0.001)$ and fatigue $(P=0.006)$, as well as functional wellbeing $(P<0.001)$ and the breast cancer subscale $(P=0.002)$ and total score $(P<0.001)$ for FACT-B (Table 4$)$.

Early complications had statistically significant adverse effects on many of the EORTC and some FACT-B subscales, with increased levels of depression according to HADS. Longer-term complications appeared to have a lesser impact, with a significant adverse effect only on body image $(P<0.001)$. Significant improvements from 3 to 12 months were noted for global quality of life, role and social functioning, fatigue, pain $(P<0.001$ for all $)$, breast symptoms $(P=0.009)$ and depression $(P=0.002)$, as well as physical and functional wellbeing, the breast cancer subscale and the total score for FACT-B $(P<0.001$ for all) (Table 4). Baseline/preoperative HRQL scores were statistically significant predictors of scores from 3 to 12 months after surgery for many of the domains investigated, and consequently were adjusted for in the regression models.

\section{Discussion}

These results confirm the independent adverse effects of early surgical complications on most aspects of HRQL over the first 12 months after surgery, which are compounded by chemotherapy. The PROMs used here did not distinguish any significant overall differences between the types of LD flap breast reconstruction with, and without PMRT. Only two of all the selected HRQL domains, pain and role functioning, were significantly worse after ALD flap reconstruction. This may be explained by poorly practised preventive measures regarding pain control and physiotherapy referral, as reported in the UK breast reconstruction audit ${ }^{3}$. These findings need to be explored further, with longer-term follow-up, in a formal clinical trial setting.

PMRT did not affect any aspect of women's quality of life in the investigated domains. The need to individualize options for immediate reconstruction when PMRT is 
anticipated is predicated on better radiation tolerability by autologous flaps ${ }^{7,25}$. No differences in PROMs were found in a single randomized trial evaluating delayed autologous flaps following PMRT in a small group of 28 patients $^{26}$. Fewer than a quarter $(n=6)$ of the studies included in systematic reviews contained patients who had received PMRT, of which five were retrospective studies with limited patient numbers ${ }^{4,11}$. Studies employing single cross-sectional analysis have shown 'excellent' or 'good' PROMs following PMRT after either immediate ALD breast reconstruction or autologous microvascular free flaps ${ }^{7,25}$. Importantly, these findings suggest there was no independent effect of PMRT on PROMs using a repeated-measures analysis, confirming a previous related analysis ${ }^{7}$. Such evidence represents a major shift in treatment recommendations and contrasts with findings from historical studies on irradiation of immediate free microvascular flaps ${ }^{9}$. The present study examined the effects of PMRT on HRQL after LDI breast reconstruction on an intention-to-treat basis and found no demonstrable effects on HRQL. However, the practice of LDI breast reconstruction would still not currently be recommended when expectation of PMRT is high ${ }^{7,9}$. None of the four prospective studies in two systematic reviews evaluated the number of women who received chemotherapy, and did not account for its potential effects on HRQL after breast reconstruction. Moreover, none of these studies analysed HRQL results in the context of multivariable regression analyses that incorporated adjuvant treatments ${ }^{4,11}$.

Women choosing immediate breast reconstruction have sociodemographic and clinical characteristics that may influence HRQL outcomes, including patient preferences and the complexity of patient choice ${ }^{27}$. The groups studied here were comparable sociodemographically, thus negating any potential differential effects on HRQL. Inherent biases on the part of patients, surgeons and healthcare professionals are inevitable confounding factors, including selection bias of ALD for those anticipated to require $\mathrm{PMRT}^{7,9}$. One of the strengths of this study is the integration of surgical complications into analyses of surgical factors on PROMs ${ }^{4,11}$. Higher level 3 complications at 3 months in this study compared with the National Mastectomy and Breast Reconstruction Audit may relate to the practice of 'clinician reporting' with stipulated levels of grading here, compared with the potential influence of 'patient recall' after 3 months in the national audit ${ }^{3}$. Furthermore, the potential for chemotherapy to impair key domains in HRQL may pertain not only to the early postoperative phase, but may persist for 12 months or longer, emphasizing the importance of integration into analyses of PROMs ${ }^{4,11}$. Patients at diagnosis of breast cancer have impaired HRQL compared with an age-matched population of women without cancer, underscoring the importance of evaluating preoperative or baseline HRQL when considering the long-term effects of breast reconstruction on most HRQL domains $^{12,18}$.

Both systematic reviews of PROMs in breast reconstruction revealed a dearth of validated breast reconstructionspecific questionnaires ${ }^{4,11}$. A phase 3 BREAST-Q questionnaire for breast reconstruction was published in 2009, and validation of a European equivalent is in progress ${ }^{28,29}$. The findings of similar effects on aspects of HRQL by type of surgery in this cohort will require further study using breast reconstruction-specific questionnaires.

Under-reporting of complications is common, despite internationally agreed methods for classification ${ }^{4,11,23}$. The UK breast reconstruction audit reported a higher than expected number of level 2 (moderate) and level 3 (major) complications (16 per cent) after similar types of breast reconstruction ${ }^{3,23}$. Indeed, up to 52 per cent of women experienced poor pain control at 3 months after breast reconstruction, compared with relatively high levels of postoperative pain (12 per cent) at 18 months $^{3}$. In the present study, the frequency of patients with level 3 complications requiring chronic treatment or reoperation was 38.1 per cent up to 3 months after surgery, and 39 per cent from 4 to 12 months, among those with complications $^{23}$. This type of information should be included in standardized informed consent procedures in the future.

The prospective longitudinal cohort design helps guide decision-making on breast reconstruction for patients and surgeons. Informed consent for breast reconstruction must include the effects of adjuvant treatments, with particular attention to such issues in younger women for whom reported preoperative HRQL scores are lower and anxiety levels correspondingly higher. The majority of studies report 12-month data only, and more prolonged follow-up to 24 and 36 months is an important intended goal of this study ${ }^{4,11}$. Clear and non-biased communication of existing clinical evidence supported by PROMs is essential ${ }^{3,4,11}$. This is the basis of standard information used in the QUEST trial (the first multicentre randomized trial of breast reconstruction within the $\mathrm{UK})^{30}$. Such attempts represent a new era in the field of breast reconstructive practice where there is a pressing need for evidence-based clinical decision-making in partnership with validated PROMs. 


\section{Collaborators}

Multicentre UK collaborations involved: M. Greenslade, K. Choudhry, A. Chaudhry, Z. Rayter, H. J. Thomson and M. Agg (Bristol); J. Mills (Institute of Cancer Research, Sutton); E. Weiler-Mithoff(Glasgow); P. McManus (Hull); S. Nicholson (York); and M. Galea (Swindon).

\section{Acknowledgements}

The authors would like to express their gratitude to Ms Margaret Agg, who was responsible for establishing the Access database for data management. They also thank the teams of breast care nurses at all of the UK collaborating centres for their hard work and dedication: Angela Nicholson, Miranda Cooper, Ingrid Fuchs, Jackie Skinner, Lorna Taylor, Lorraine Peall, Naomi Day (Bristol); Joanna Rowley (Cambridge); Elaine Gullakson (East Yorkshire); Claire South, Caroline Spreng (Glasgow); Jan Dodge (Swindon); and Debbie Nicholson (York).

The study received operating grants from BUPA Charitable Giving and Abreast Cancer Research, Inamed Aesthetics (Allergan UK), and the University Foundation Hospitals of Bristol NHS Above and Beyond Charitable Trustees. Z.E.W. is a founding trustee of Abreast Cancer Research, and is also a member of the European Faculty of Allergan UK. J.H. is funded from a core grant to ICR-CTSU from Cancer Research UK. The funders had no role in the study design, collection, analysis or interpretation of data, or in the writing of the report.

Disclosure: The authors declare no other conflict of interest.

\section{References}

1 Maddams J, Moller H, Devane C. Cancer Prevalence in the UK, 2008. Thames Cancer Registry and Macmillan Cancer Support: London, 2008.

2 Rowland JH, Hewitt M, Ganz PA. Cancer survivorship: a new challenge in delivering quality cancer care. 7 Clin Oncol 2006; 24: 5101-5104.

3 NHS Information Centre. National Mastectomy and Breast Reconstruction Audit 2009. http://www.ic.nhs.uk/mbr [accessed 30 November 2011].

4 Winters ZE, Benson JR, Pusic AL. A systematic review of the clinical evidence to guide treatment recommendations in breast reconstruction based on patient-reported outcome measures and health-related quality of life. Ann Surg 2010; 252: 929-942.

5 Langenhoff BS, Krabbe PF, Wobbes T, Ruers TJ. Quality of life as an outcome measure in surgical oncology. Br 7 Surg 2001; 88: 643-652.
6 Mayor S. NICE updates guidance on medical and surgical treatment for early and advanced breast cancer. BMF 2009; 338: b815.

7 Thomson HJ, Potter S, Greenwood RJ, Bahl A, Barker J, Cawthorn SJ et al. A prospective longitudinal study of cosmetic outcome in immediate latissimus dorsi breast reconstruction and the influence of radiotherapy. Ann Surg Oncol 2008; 15: 1081-1091.

8 Harris Jr. Radiation therapy for invasive breast cancer: not just for local control. 7 Clin Oncol 2005; 23: 1607-1608.

9 Javaid M, Song F, Leinster S, Dickson MG, James NK. Radiation effects on the cosmetic outcomes of immediate and delayed autologous breast reconstruction: an argument about timing. F Plast Reconstr Aesthet Surg 2006; 59: 16-26.

10 Blazeby JM, Avery K, Sprangers M, Pikhart H, Fayers P, Donovan J. Health-related quality of life measurement in randomized clinical trials in surgical oncology. 7 Clin Oncol 2006; 24: 3178-3186.

11 Lee C, Sunu C, Pignone M. Patient-reported outcomes of breast reconstruction after mastectomy: a systematic review. 7 Am Coll Surg 2009; 209: 123-133.

12 Goodwin PJ, Black JT, Bordeleau LJ, Ganz PA. Health-related quality-of-life measurement in randomized clinical trials in breast cancer - taking stock. 7 Natl Cancer Inst 2003; 95: 263-281.

13 Edge SB, Compton CC. The American Joint Committee on Cancer: the 7th edition of the AJCC cancer staging manual and the future of TNM. Ann Surg Oncol 2010; 17: 1471-1474.

14 Overgaard M, Hansen PS, Overgaard J, Rose C, Andersson M, Bach F et al. Postoperative radiotherapy in high-risk premenopausal women with breast cancer who receive adjuvant chemotherapy. Danish Breast Cancer Cooperative Group 82b Trial. N Engl f Med 1997; 337: 949-955.

15 Ragaz J, Jackson SM, Le N, Plenderleith IH, Spinelli JJ, Basco VE et al. Adjuvant radiotherapy and chemotherapy in node-positive premenopausal women with breast cancer. NEngl 7 Med 1997; 337: 956-962.

16 McPherson K. Do patients' preferences matter? BM7 2009; 338: $59-60$.

17 Garratt A, Schmidt L, Mackintosh A, Fitzpatrick R. Quality of life measurement: bibliographic study of patient assessed health outcome measures. BM7 2002; 324: 1417.

18 Efficace F, Bottomley A, Osoba D, Gotay C, Flechtner H, D'Haese $\mathrm{S}$ et al. Beyond the development of health-related quality-of-life (HRQOL) measures: a checklist for evaluating HRQOL outcomes in cancer clinical trials - does HRQOL evaluation in prostate cancer research inform clinical decision making? 7 Clin Oncol 2003; 21: 3502-3511.

19 Sprangers MA, Groenvold M, Arraras JI, Franklin J, te Velde A, Muller M et al. The European Organization for Research and Treatment of Cancer breast cancer-specific quality-of-life questionnaire module: first results from a three-country field study. 7 Clin Oncol 1996; 14: 2756-2768. 
20 Hopwood P, Fletcher I, Lee A, Al Ghazal S. A body image scale for use with cancer patients. Eur 7 Cancer 2001; 37 : 189-197.

21 Zigmond AS, Snaith RP. The hospital anxiety and depression scale. Acta Psychiatr Scand 1983; 67: 361-370.

22 Brady MJ, Cella DF, Mo F, Bonomi AE, Tulsky DS, Lloyd SR et al. Reliability and validity of the Functional Assessment of Cancer Therapy-Breast quality-of-life instrument. 7 Clin Oncol 1997; 15: 974-986.

23 Dindo D, Demartines N, Clavien PA. Classification of surgical complications: a new proposal with evaluation in a cohort of 6336 patients and results of a survey. Ann Surg 2004; 240: 205-213.

24 Association of Breast Surgery at BASO 2009. Surgical guidelines for the management of breast cancer. Eur 7 Surg Oncol 2009; 35(Suppl 1): 1-22.

25 Chatterjee JS, Lee A, Anderson W, Baker L, Stevenson JH, Dewar JA et al. Effect of postoperative radiotherapy on autologous deep inferior epigastric perforator flap volume after immediate breast reconstruction. Br 7 Surg 2009; 96: $1135-1140$

26 Brandberg Y, Malm M, Blomqvist L. A prospective and randomized study, 'SVEA,' comparing effects of three methods for delayed breast reconstruction on quality of life, patient-defined problem areas of life, and cosmetic result. Plast Reconstr Surg 2000; 105: 66-74.

27 Christian CK, Niland J, Edge SB, Ottesen RA, Hughes ME, Theriault $\mathrm{R}$ et al. A multi-institutional analysis of the socioeconomic determinants of breast reconstruction: a study of the National Comprehensive Cancer Network. Ann Surg 2006; 243: 241-249.

28 Pusic AL, Klassen AF, Scott AM, Klok JA, Cordeiro PG, Cano SJ. Development of a new patient-reported outcome measure for breast surgery: the BREAST-Q. Plast Reconstr Surg 2009; 124: 345-353.

29 Winters ZE, Thomson HJ. Assessing the clinical effectiveness of breast reconstruction through patient-reported outcome measures. Br 7 Surg 2011; 98 : $323-325$.

30 Winters ZE, Mills J, Kilburn L, Horne R, Kapari M, Bliss J. The QUEST Trial: a multi-centre randomised trial to assess the impact of the type and timing of breast reconstruction on quality of life following mastectomy. EFC 2010; 8(Suppl): 142.

\section{Snapshot Quiz}

Answer

\section{Snapshot Quiz 13/23}

At laparotomy a torted and infarcted gallbladder was found, and a cholecystectomy was therefore performed. The patient made an uneventful recovery. The authors advise that torsion of the gallbladder should be considered in any individual with sudden-onset upper abdominal pain and no signs of toxaemia. 